
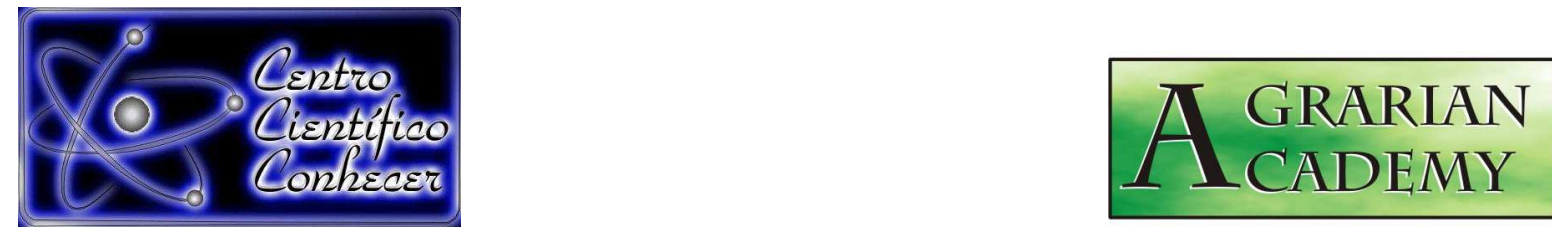

\title{
ABUNDÂNCIA DE INVERTEBRADOS DE SOLO ENCONTRADOS EM DIFERENTES AMBIENTES NA UNIVERSIDADE FEDERAL DA FRONTEIRA SUL/ Campus CHAPECÓ-SC
}

\author{
Camilla Jacques ${ }^{1}$, Dannyelle Cristine Orsolin de Morais ${ }^{2}$, Siumar Pedro Tironi ${ }^{3}$, \\ André Luiz Radunz ${ }^{4}$, Marco Aurélio Tramontin ${ }^{5}$ \\ ${ }^{1}$ Engenheira Agrônoma, Graduada em Agronomia, Universidade Federal da \\ Fronteira Sul \\ ${ }^{2}$ Graduanda em Agronomia, Universidade Federal da Fronteira Sul \\ ${ }_{3}$ Professor Doutor, Universidade Federal da Fronteira Sul \\ ${ }^{4}$ Professor Doutor, Universidade Federal da Fronteira Sul \\ ${ }^{5}$ Professor Doutor, Universidade Federal da Fronteira Sul \\ (marco.silva@uffs.edu.br) Chapecó-Brasil
}

Recebido em: 30/11/2017 - Aprovado em: 15/12/2017 - Publicado em: 31/12/2017 DOI: 10.18677/Agrarian Academy 2017b1

\begin{abstract}
RESUMO
O Brasil abrange em torno de 15 a $20 \%$ de todas as espécies existentes, mas são escassos os conhecimentos sobre essa biota. A Classe Insecta destaca-se com maior número de indivíduos. Dessa forma, objetivou-se realizar um estudo de levantamento sobre a fauna do solo em distintos lugares. A pesquisa foi efetuada na Universidade Federal da Fronteira Sul, Campus Chapecó. Os distintos lugares foram: área de pinus (1), eucalipto (2), lavoura (3), área em sucessão inicial (4) e floresta nativa (5). A coleta dos invertebrados teve duração de 50 horas, através do uso de armadilhas do tipo "Pitfall", com líquido composto por álcool etílico hidratado $70 \%$ e detergente, sem atrativos. A instalação das armadilhas ocorreu em 25/05/2015 e posteriormente o recolhimento das mesmas. Após, realizou-se a separação e classificação taxonômica das ordens com auxilio de estereomicroscópio. Assim sendo, verifica-se que os locais de coleta apresentam insetos das seguintes ordens: Araneae $(4,97 \%)$, Blattodea $(0,25 \%)$, Dermaptera $(0,46 \%)$, Diptera $(2,79 \%)$, Hemiptera $(0,66 \%)$, Odonata $(0,05 \%)$, e Thysanura $(0,10 \%)$. As áreas que detém os maiores números de animais são as que apresentaram maior diversidade de plantas; os monocultivos apresentaram menor abundância e riqueza de invertebrados; os insetos da ordem Hymenoptera foram os mais encontrados nos cinco locais de coleta.
\end{abstract}

PALAVRAS-CHAVE: Fauna do solo, Insetos, Pitfall. 


\title{
ABUNDANCE OF SOIL INVERTEBRATES FOUND IN DIFFERENT ENVIRONMENTS AT THE FEDERAL UNIVERSITY OF FRONTEIRA SUL / Campus CHAPECÓ-SC
}

\begin{abstract}
Brazil covers around 15 to $20 \%$ of all existing species, but knowledge about this biota is scarce. The Insecta Class stands out with more individuals. Thus, a study was carried out to survey the soil fauna in different places. The research was carried out at the Federal University of Fronteira Sul. The different sites were: pinus (1), eucalyptus (2), crop (3), initial succession area (4) and native forest (5). The collection of the invertebrates lasted 50 hours, through the use of "Pitfall" trap, with alcohol $70 \%$ and detergent, without attractive. The installation of the traps occurred on $05 / 25 / 2015$ and later collect of insects. After, the sorting and taxonomic classification of the orders with stereomicroscope. Therefore, it is verified that the collection sites present insects of the following ordens: Araneae $(4,97 \%)$, Blattodea $(0,25 \%)$, Dermaptera $(0,46 \%)$, Diptera $(2,79 \%)$, Hemiptera $(0,66 \%)$, Odonata $(0,05 \%)$, e Thysanura $(0,10 \%)$. The areas that had the most animals were those that presented the greatest diversity of plants; monocultures presented lower abundance and richness of invertebrates; the insects of the order Hymenoptera were the most found in the five collection sites.
\end{abstract}

KEYWORDS: Soil fauna, Insects, Pitfall

\section{INTRODUÇÃO}

O Brasil é considerado um dos países com maior biodiversidade do mundo, apresentando as denominadas Unidades de Conservação (UCs), com objetivo de preservar ao máximo a diversidade dos ecossistemas e espécies associadas (TORRES ; OLIVEIRA, 2012). A biodiversidade é definida pela Convenção sobre Diversidade Biológica (CBD- Convention on Biological Diversity) como a variabilidade de organismos vivos de todas as origens e ambientes, compreendendo ainda a diversidade de espécies, entre espécies e de ecossistemas (CBD, 1994). Diversos taxa são conhecidos para serem utilizados como bioindicadores de integridade ecológica ou de endemismo (OLIVEIRA ; TIDON, 2014).

Os efeitos causados pelos animais do solo sobre as plantas são indiretos, uma vez que alteram as características edáficas, como $\circ \mathrm{pH}$, concentração de nutrientes disponíveis, nível de pressão de insetos-praga e doenças, atividade de micro-organismos, competição, toxidez por alumínios, além da aeração e disponibilidade de água (PARRON et al., 2015).

Os insetos, também conhecidos como hexápodes são animais metazoários de simetria bilateral cujo corpo é dividido em três regiões: cabeça, tórax e abdômen, possuem três pares de pernas, podendo ou não apresentar asas e aparelho bucal ectognato (AHID, 2009). O êxito dos insetos como grupo que vem sobrevivendo a cerca de 300 milhões de anos é devido ao fato de possuírem pelo menos seis principais vantagens, na luta incessante pela sobrevivência: capacidade de voo, adaptabilidade, exoesqueleto, pequeno tamanho, metamorfose e tipo especializado de reprodução (FARIAS, 2003).

O monitoramento da diversidade dos grupos da fauna edáfica permite compreender a funcionalidade desses organismos e a complexidade ecológica dessas comunidades (JOUQUET et al., 2014). Assim, objetivou-se quantificar a 
incidência de indivíduos em cinco diferentes áreas com predominância de pinus (1), de eucalipto (2), de lavoura (3), de sucessão inicial (4) e de floresta nativa (5).

\section{MATERIAL E MÉTODOS}

O estudo foi desenvolvido no Campus da Universidade Federal da Fronteira Sul (UFFS) nas seguintes coordenadas (27은 $\left.53,75^{\prime \prime} S, 52^{\circ} 42^{\prime} 23,85^{\prime \prime} \mathrm{O}\right)$, em cinco

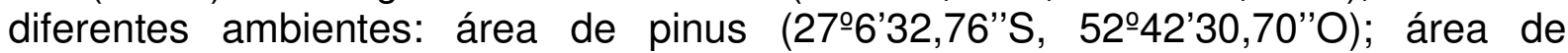

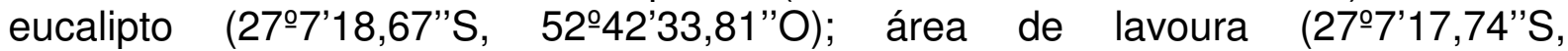

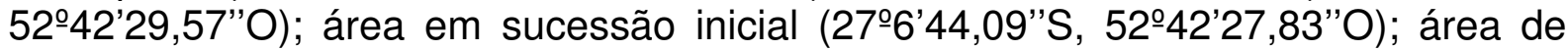

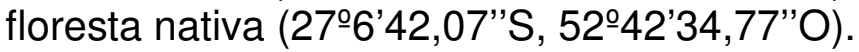

Foram instaladas cinco armadilhas em linha contínua com quatro repetições a um metro de distância uma da outra em cada local amostrado, e assim um total de 20 amostras em cada área (100 amostras ao todo). Para a escolha da localização das armadilhas, realizou-se sorteio aleatório por meio do software Excel. Nas áreas de Eucalipto e Pinus, a instalação foi realizada através de um transecto por serem áreas muito pequenas em largura. Nas demais áreas, as armadilhas foram instaladas aleatoriamente segundo o sorteio realizado.

Foram utilizadas armadilhas tipo "Pitfall", utilizando-se copos plásticos de 100 $\mathrm{mL}$, com aproximadamente $10 \mathrm{~cm}$ de profundidade e $6 \mathrm{~cm}$ de diâmetro. Como solução líquida conservante foi utilizado $50 \mathrm{~mL}$ de álcool etílico hidratado $70 \%$, com três gotas de detergente comum. As armadilhas do tipo "Pitfall" foram instaladas no dia 25/05/2015 as $14 \mathrm{~h} 00 \mathrm{~min}$, e retiradas no dia 27/05/2015 as $16 \mathrm{~h} 00 \mathrm{~min}$ horas, permanecendo no campo por aproximadamente 50 horas.

Os espécimes capturados nas armadilhas, depois de armazenados, foram levados ao Laboratório de Botânica, Ecologia e Entomologia da UFFS, e separados com auxilio de estereomicroscópio para determinação da Ordem taxonômica. O Índice de Similaridade foi realizado através do Software Past, versão 3.11 (Hammer et al., 2001), através de distanciamento do tipo Bray-Curtis.

\section{RESULTADOS E DISCUSSÃO}

Com base nos dados da Figura 1, verificou-se que os locais com maior número de indivíduos foram da área em sucessão inicial, com 599 indivíduos (30,4\%), e a de Lavoura com 560 indivíduos (28,4\%). Em nível intermediário, ficaram as áreas de Eucalipto, com 282 indivíduos (14,3\%), e de Floresta nativa, com 330 indivíduos (16,7\%).

Em contrapartida, a área que houve menor incidência de indivíduos, foi a de Pinus, com apenas 201 (10,2\%). Assim, foi obtido um total de 1.972 indivíduos em 11 diferentes grupos sendo eles: Araneae, Blattodea, Coleoptera, Collembola, Dermaptera, Diptera, Hemiptera, Hymenoptera, Odonata, Orthoptera e Thysanura. 


\section{Percentagem de indivíduos por área}

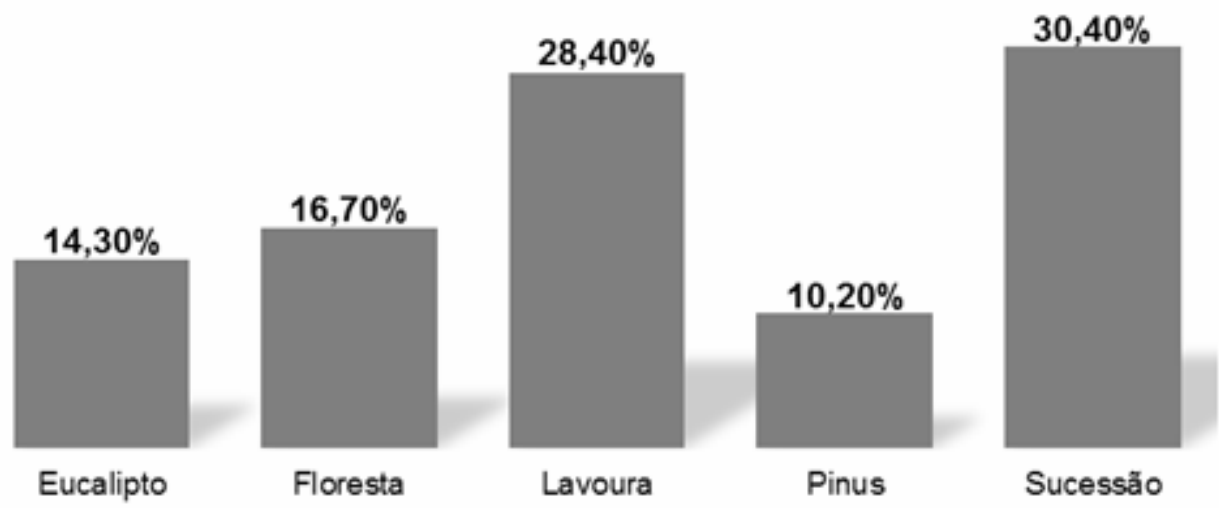

FIGURA 1 - Percentual de indivíduos coletados em cada área de coleta.

Fonte: Elaborado pelos autores.

Conforme a Tabela 1, observa-se o número de indivíduos capturados em cada uma das 11 ordens amostradas e o local onde estes indivíduos foram coletados, evidenciando a diferença em abundância de invertebrados em cada área distinta.

TABELA 1 - Número de indivíduos de cada ordem capturados em cada uma das cinco áreas amostradas.

\begin{tabular}{cccccc}
\hline Ordem & Eucalipto & Floresta & Lavoura & Pinus & sucessão inicial \\
\hline Araneae & 8 & 25 & 10 & 9 & 46 \\
Blattodea & 0 & 0 & 0 & 5 & 0 \\
Coleoptera & 2 & 8 & 64 & 1 & 30 \\
Collembola & 50 & 58 & 49 & 56 & 98 \\
Dermaptera & 3 & 0 & 2 & 0 & 4 \\
Diptera & 7 & 19 & 21 & 4 & 4 \\
Hemiptera & 1 & 1 & 7 & 1 & 3 \\
Hymenoptera & 201 & 214 & 233 & 122 & 406 \\
Odonata & 0 & 0 & 0 & 0 & 1 \\
Orthoptera & 8 & 5 & 174 & 3 & 7 \\
Thysanura & 2 & 0 & 0 & 0 & 0 \\
\hline Total & 282 & 330 & 560 & 201 & 599 \\
\hline
\end{tabular}

$\mathrm{Na}$ Figura 2, destacam-se os himenópteros capturados em relação às outras ordens. Assim, esses exemplares da Ordem Hymenoptera corresponderam a 59,6\% de todos os indivíduos amostrados, e $40,4 \%$ dos indivíduos distribuíram-se em outros grupos. Isso é explicado, conforme Fleck et al., (2015) que as formigas constituem o maior grupo de insetos sociais, sendo amplamente distribuídas geograficamente, e são encontradas em áreas que vão desde regiões subpolares até o Equador, bem como em todas as ilhas oceânicas, exceto nos polos e nos mares, sendo mais abundantes em locais de clima tropical. 
A Ordem Hymenoptera possui maior destaque principalmente em relação aos exemplares da família Formicidae, pois são predominantes na maioria dos ecossistemas terrestres, no qual sua abundância relaciona-se à quantidade de alimento disponível e sítios de nidificação (ZARDO et al., 2015). Valores semelhantes foram coletados e analisados por Libera et al., (2017), quando encontraram aproximadamente $57 \%$ de exemplares da Ordem Hymenoptera, e destes, $40 \%$ eram da família Formicidae na cultura da abobrinha.

\section{Ordem Hymenoptera}

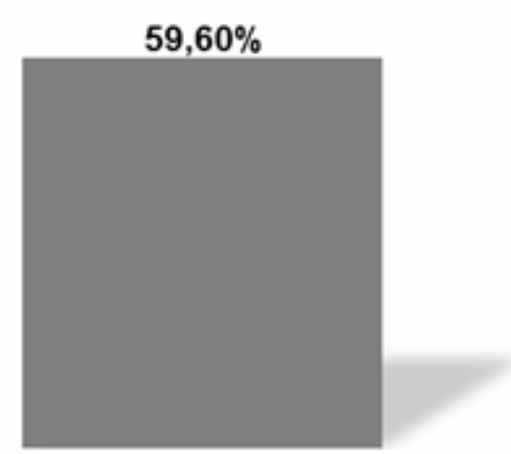

Hymenoptera

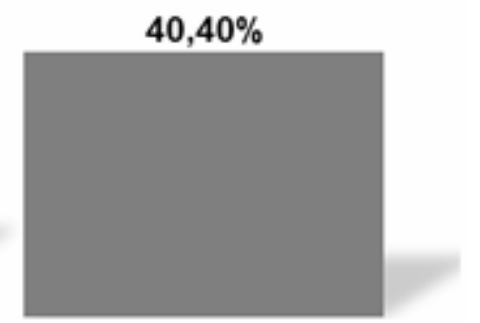

Outros

\section{FIGURA 2 - Percentual de himenópteros capturados em relação aos indivíduos das demais ordens.}

Fonte: Elaborado pelos autores

$\mathrm{Na}$ área de Eucalipto (Figura 3), obtiveram-se nove diferentes grupos de animais, sendo os himenópteros (201) e os colêmbolos (50) em maior número. Em comparação ao trabalho de Silveira Neto et al., (1995) apresentou reduzido número de indivíduos, porém, assemelhou-se com dados apresentados por Forsthofer \& Athayde-Filho (2012) que justificam que este fato se relaciona às diferenças de características ambientais das áreas amostradas, e também aos métodos de coleta realizados.

\section{Eucalipto}

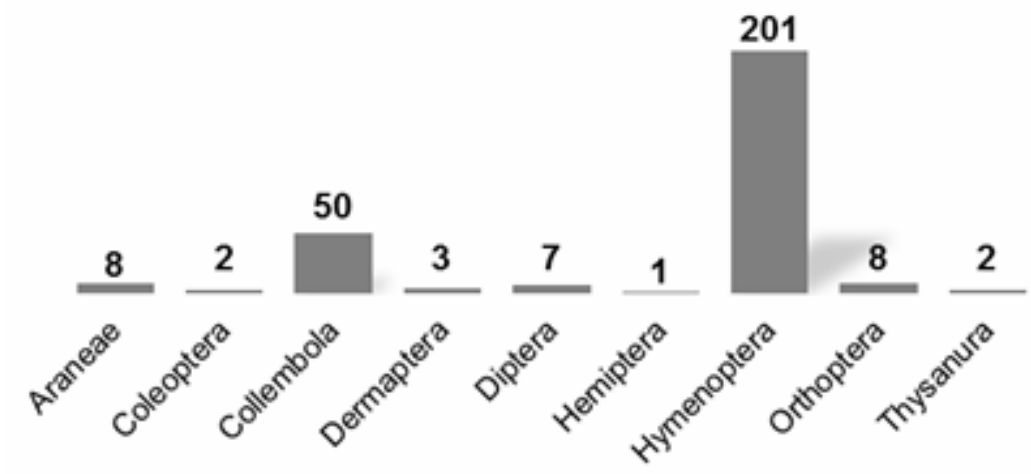

FIGURA 3 - Representação da abundância de indivíduos amostrados na área de Eucalipto.

Fonte: Elaborado pelos autores. 
$\mathrm{Na}$ área de Floresta (Figura 4), verificaram-se sete diferentes grupos, e da mesma maneira que na área de Pinus, os himenópteros (122) e os colêmbolos (58) em maior número. Obtiveram-se também números significativos de aracnídeos (25) e dípteros (19). A diversidade da fauna nativa relaciona-se com os agroecossistemas devido à variedade de recursos e hábitats que o sistema soloserrapilheira fornece, favorecendo condições climáticas aos diversos grupos funcionais associados (DANTAS et al., 2012).

De acordo com Laroca (1995), os estudos sobre levantamentos populacionais de invertebrados são ferramentas importantes para conservação da biodiversidade, destacando-se o grupo de insetos polinizadores, devido a sua função desempenhada para sucesso reprodutivo das plantas, sejam elas cultivadas ou não, e estas plantas por sua vez são fontes de recursos alimentares para as demais espécies dependentes.

\section{Floresta}

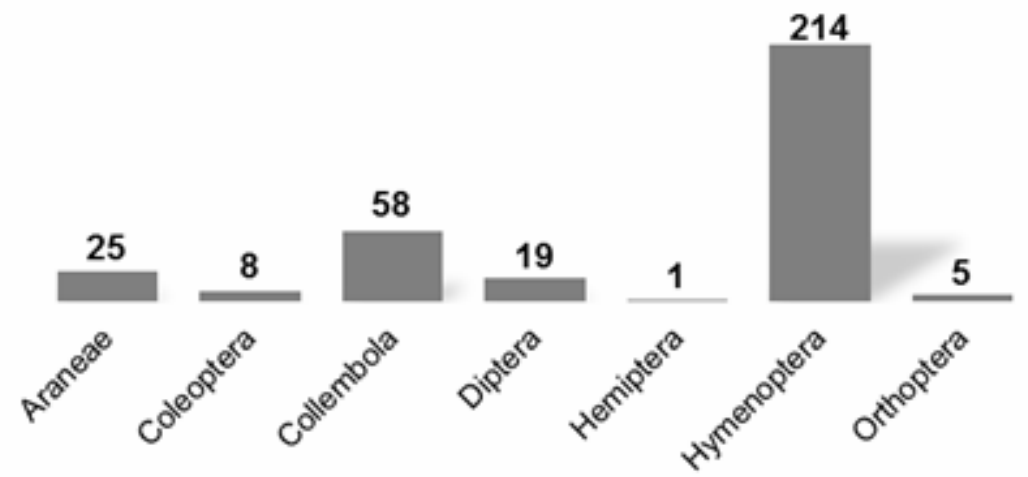

FIGURA 4 - Representação da abundância de indivíduos amostrados na área de Floresta.

Fonte: Elaborado pelos autores.

$\mathrm{Na}$ área de Lavoura (Figura 5), coletaram-se um total de oito diferentes ordens. Os himenópteros (233), ortópteros (172) e coleópteros (64) foram os grupos com maior abundância de indivíduos. Esta área foi a segunda maior área em número de indivíduos, ficando atrás apenas da área em sucessão inicial. Isso ocorreu devido ao elevado número de ortópteros amostrados na área, conforme é explicado na Figura 5. 


\section{Lavoura}

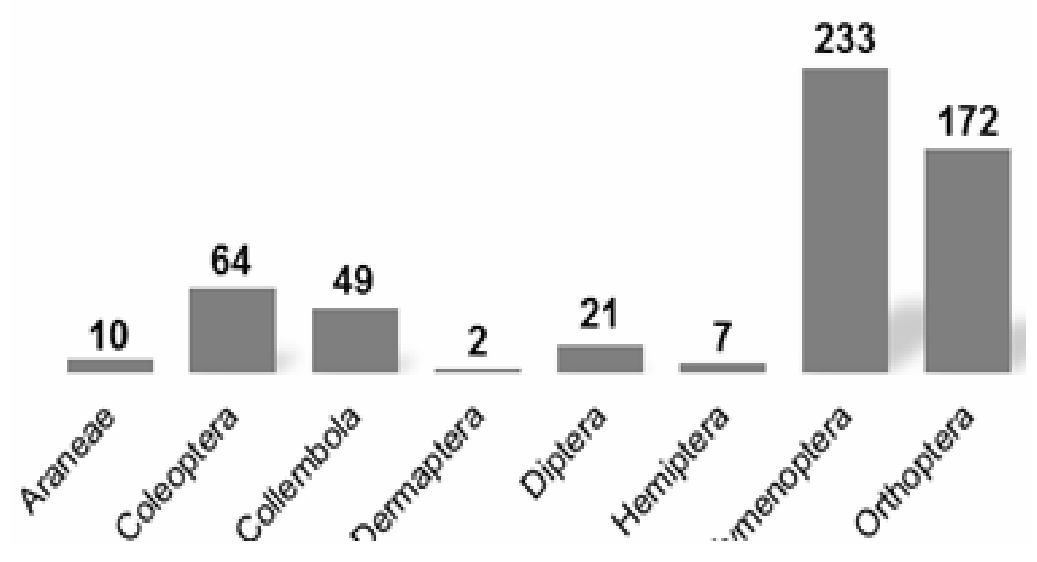

FIGURA 5 - Representação da abundância de indivíduos amostrados na área de Lavoura.

Fonte: Elaborado pelos autores

Os coleópteros apresentaram número mais significativo nesta área, sendo que nas demais não houve dados expressivos. A Ordem Coleoptera abrange diversas espécies distribuídas em diferentes ecossistemas terrestres, apresentam diversos comportamentos podendo ser pragas, inimigos naturais, decompositores de matéria orgânica e polinizadores (LIMA et al., 2013).

Em relação à ordem Orthoptera (Figura 6), observou-se uma abundância significantemente maior na área de Lavoura (88\%), quando comparada às demais áreas (média de 3\%). Conforme estudos de Guerra et al., (2012), a abundância de ortópteros em lavouras é significativa, isto pelo fato de serem considerados como insetos-praga agrícolas de várias culturas.

\section{Ordem Orthoptera}

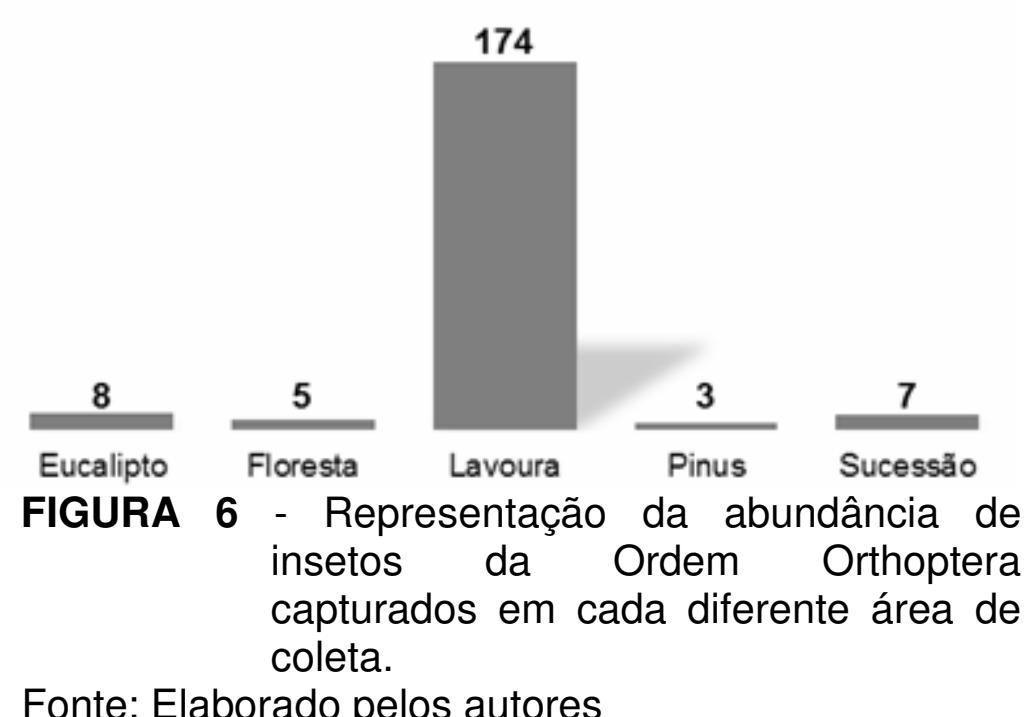


Na área de Pinus (Figura 7), obtiveram-se oito diferentes ordens, sendo os colêmbolos (56) e os himenópteros (122) os mais significativos. Os plantios florestais, por serem monoculturas, simplificam o ambiente em que são implantados, da mesma forma como acontece em monoculturas agrícolas e pastagens (GONTIJO,2017). Observou-se assim, uma relação entre a área de pinus ser a mais homogênea e também ser a área com menor diversidade de invertebrados e número de indivíduos.

\section{Pinus}

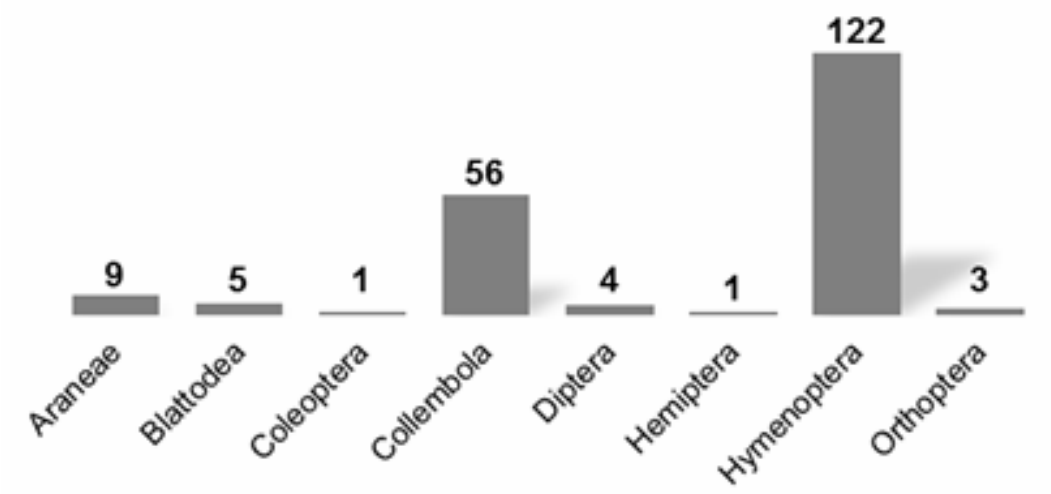

FIGURA 7 - Representação da abundância de indivíduos amostrados na área de Pinus.

Fonte: Elaborado pelos autores

$\mathrm{Na}$ área de sucessão inicial obtiveram-se nove diferentes ordens. Assim, os números mais significativos de indivíduos foram os himenópteros (406), colêmbolos (98), aracnídeos (46) e coleópteros (30) (Figura 8). Como a área apresenta uma grande biodiversidade de espécies de plantas, isso confirma a hipótese de Risch et al. (1983), que afirmam que áreas com cultivos diversificados apresentam maior diversidade de insetos. Os himenópteros foram na totalidade compostos por formigas, isso ocorre, devido ao modelo das armadilhas de solo ("Pitfall"), e também caracterizado pelo grande número de formigueiros no ambiente.

\section{Sucessão Inicial}

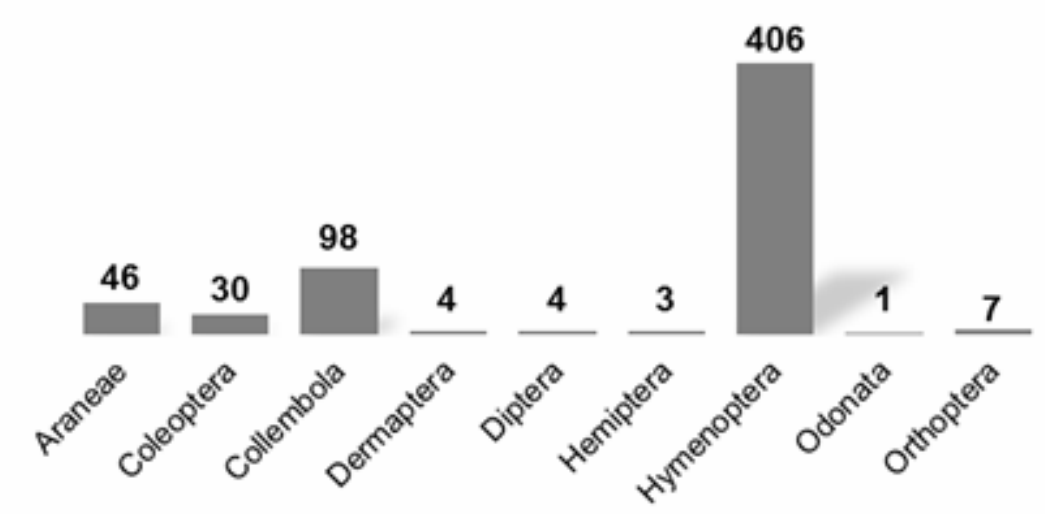

FIGURA 8 - Representação da abundância de indivíduos amostrados na área em sucessão inicial.

Fonte: Elaborado pelos autores 


\section{CONCLUSÕES}

1 - Os invertebrados capturados com armadilhas do tipo "Pitfall" nos locais observados são pertencentes aos grupos de: aracnídeos, blatódeos, coleópteros, colêmbolos, dermápteros, dípteros, hemípteros, himenópteros, odonatos, ortópteros e tisanuros;

2 - As amostras com números elevados de indivíduos invertebrados foram pertencentes aos locais de área em sucessão inicial (599) e de Floresta (560);

3 - A área com menor abundância de invertebrados foi a de Pinus (201);

4- As áreas de Pinus e Lavoura demonstraram menor diversidade de invertebrados;

5 - A Ordem Hymenoptera foi a mais abundante em todas as amostras, com 1.176 indivíduos coletados.

\section{REFERÊNCIAS}

AHID, S.M.M. Apostila Didática em Entomologia Veterinária. Mossoró: UFERSA, 2009. $80 \mathrm{p}$.

CBD- Convention on biological diversity. Rio de Janeiro, Brasil. 1994.

DANTAS, J. O., SANTOS, M. J. C., SANTOS, F. R., PEREIRA, T. P. B., OLIVEIRA, A. V. S., ARAÚJO, C. C., RITA, M. R. Levantamento da entomofauna associada em sistema agroflorestal. Scientia Plena. 2012. 8 p.

FARIAS, P. R. S. Manual de Entomologia Geral. Amazonas: UFRA- Universidade Federal Rural da Amazônia, 2003. 142 p.

FLECK, M. D.; CANTARELLI, D. B; GRANZOTTO, F. Registro de novas espécies de formigas (Hymenoptera: Formicidae) no estado do Rio Grande do Sul. Ciência Florestal, v. 25, n. 2, p. 491-499, 2015.

FORSTHOFER, M.; ATHAYDE-FILHO, F. P. A. Florística e aspectos ecológicos de samambaias e licófitas ao longo do córrego Cachoeirinha, Nova XavantinaMT. Pesquisas, Botânica, v. 63, p. 149-164, 2012.

Hammer, Ø., Harper, D.A.T., Ryan, P.D. PAST: Paleontological statistics software package for education and data analysis. Palaeontologia Electronica 4(1): 9pp. 2001.

GONTIJO, B. M. G. Implicações do plantio generalizado de Eucaliptus no empobrecimento social e da biodiversidade do alto/médio Jequitinhonha-MG. Boletim Paulista de Geografia. 22 p. 2017.

GUERRA, W.D. OLIVEIRA, P. C. de; PUJOL-LUZ, J.R. Gafanhotos (Orthoptera, Acridoidea) em áreas de Cerrados e Lavouras na Chapada dos Parecis, Estado do Mato Grosso, Brasil. Revista Brasileira de Entomologia, v. 56, n. 2, p. 228-239 2012.

JOUQUET, P.; BLANCHART, E.; CAPOWIEZ, Y. Utilization of earthworms and AGRARIAN ACADEMY, Centro Científico Conhecer - Goiânia, v.4, n.8; p.9 2017 
termites for the restoration of ecosystem functioning. Applied Soil Ecology, Pretty, v. $73, \quad$ n. 1, p. 34-40, 2014.Disponível em: http://www.sciencedirect.com/science/article/pii/S0929139313002072. Doi: https://doi.org/10.1016/j.apsoil.2013.08.004

LAROCA, S. Ecologia: princípios e métodos. Petrópolis: Vozes, 1995. 197 p.

LIBERA, D.D., TIRONI, S.P., RADUNZ, A.L., TRAMONTIN, M.A. Diversidade populacional de artrópodes na cultura da abobrinha utilizando armadilhas do tipo Moerick e Pitfall. Agrarian Academy, Goiânia, v.4, n.7, p. 176-184, 2017. Disponível em:

http://www.conhecer.org.br/Agrarian\%20Academy/2017a/diversidade\%20populacion al.pdf. DOI: 10.18677/Agrarian_Academy_2017a16

LIMA, M. G. A.; SILVA, R. P. A.; SOUSA, M. D. F.; COSTA, E. M. Diversidade de Scarabaeinae (Coleoptera: Scarabaeidae) no parque botânico do Ceará, CaucaiaCE, Brasil. Revista Agroambiente On-line, v. 7, n. 1, p. 89-94, 2013.

OLIVEIRA, S. C. F., TIDON, R. Biodiversity surrogates: funcionality and limitations. Oecologia Australis. v 4. p. 474-482. 2014.

PARRON, L. M. GARCIA, J. R. OLIVEIRA, E. B. BROWN, G. G. PRADO, R. B. Serviços ambientais em Sistemas Agrícolas e Florestais do Bioma Mata Atlântica. Empresa Brasileira de Pesquisas Agropecuárias (EMBRAPA). Brasília, DF, p.10154, 2015.

RISCH, S.J.; ANDOW, D.; ALTIERI, M.A. Agroecosystem diversity and pest control: data, tentative conclusion and new research directions. Environmental Entomology, v.12, p.625-629, 1983. Disponível em: <https://academic.oup.com/ee/articleabstract/12/3/625/411353? redirectedFrom=fullte xt> Doi: https://doi.org/10.1093/ee/12.3.625

SILVEIRA NETO, S., MONTEIRO, R. C., ZUCCHI, R. A., MORAES, R. C. B. Uso da análise faunística de insetos na avaliação do impacto ambiental. Scientia Agricola, Piracicaba, v. 52, n. 1, p. 9-15, 1995 . Disponível em:< http://www.scielo.br/scielo.php?pid=S0103-

90161995000100003\&script=sci_abstract\&tIng=pt $>$.

Doi: http://dx.doi.org/10.1590/S0103-90161995000100003.

TORRES, D. F; OLIVEIRA, E. S. Percepção ambiental: instrumento para educação ambiental em unidades de conservação. REMEA-Revista Eletrônica do Mestrado em Educação Ambiental, v. 21. 9p. 2012.

ZARDO, D. C., CARNEIRO, Â. P., DE LIMA, L. G., \& DOS SANTOS FILHO, M. Comunidade de artrópodes associada a serrapilheira de cerrado e mata de galeria, na Estação Ecológica Serra das Araras-Mato Grosso, Brasil. Revista ReBraM, 13(2), p 105-113. 2015. 